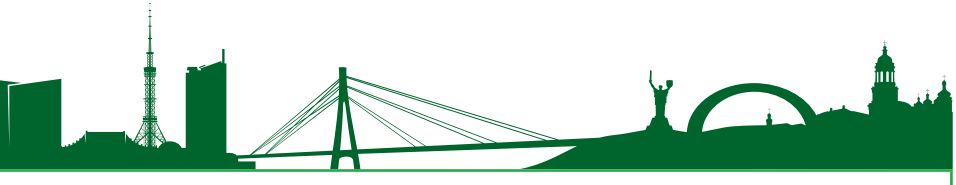

Doi: https://doi.org/10.33644/scienceandconstruction.v21i3.113

УДК 624.014

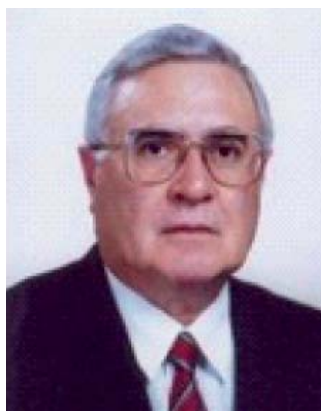

СЕЙФУЛААЕВ ХАНААР КУРБАН ОГАЫ

Д-р технических наук, проф., зав. отделом Азербайджанского научно-исследовательского института строительства и архитектуры,

г. Баку, Азербайджан

тел.: +994506200710

e-mail:xanlar.seyfullayev@mail.ru

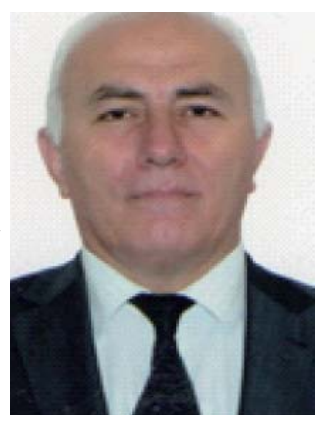

ГАРАЕВ АБДЫ НАЗИМ ОГАЫ

Канд. технических наук, директор Азербайджанс-кого НИИ строительства и архитектуры, г. Баку, Азербайджан тел.: +994502152497

e-mail: azimeti.nti@gmail.com

\title{
УСОВЕРШЕНСТВОВАННАЯ МЕТОДИКА РАСЧЕТА ЖЕЛЕЗОБЕТОННЫХ ЭЛЕМЕНТОВ НА ПРОЧНОСТЬ И СЕЙСМОСТОЙКОСТЬ С УЧЕТОМ ТРЕБОВАНИЙ ЕВРОКОДОВ
}

\section{АННОТАЦИЯ}

В работе рассматривается сравнение национального норматива по железобетону AzDTN 2.16-1 и одноименного норматива Франции BAEL-85, выявлены некоторые нестыковки результатов расчетов и изучены их причины. Приводятся пути устранения нестыковки полученных решений.

К нестыковкам двух нормативных документов относятся различия во взглядах на предельное состояние железобетонных элементов и результаты расчета с применением этих различных предельных состояний, использование нелинейной деформационной модели, основой которой является гипотеза плоских сечений и диаграммы состояния бетона, линеаризации решения задачи путем замены криволинейных форм диаграмм состояния бетона кусочно-линейными формами, решение задачи длительной прочности бетона с введением понятия о нисходящей ветви криволинейной диаграммы деформации бетона и другие проблемы, характеризующие свойства бетона сжатой зоны.

В работе рассматривается статическая, геометрическая и физическая стороны упругопластического изгиба железобетонных элементов. В основу нелинейной деформационной модели, при расчете железобетонных конструкций, положены предельные состояния железобетонных элементов, описываемые прямолинейными диаграммами деформации и кусочно-линейными диаграммами состояния бетона.

Решение задачи изгибаемых элементов, на основе нелинейной деформационной модели, при кусочно-линейных диаграммах состояния бетона, сводится к решению линейных уравнений статики, при соблюдении гипотезы плоских сечений и аналитических выражений двухлинейной диаграммы состояния бетона.

По требованию Еврокода в расчетах предлагается использовать криволинейную диаграмму с ниспадающей ветвью, отражающую поведение бетона при сжатии. Одновременно, согласно AzDTN 2.16-1, кроме кусочно-линейных диаграмм, в расчетах состояние бетона может быть использована криволинейная диаграмма с ниспадающей ветвью. При этом, должны быть обозначены основные параметрические точки диаграмм (максимальное напряжение и соответствующие деформации, граничные значения и т.д.).

Результаты исследования показывают, что при $R_{b l} / R_{b} \geq 0.85$ влиянием длительной прочности бетона в практических расчетах можно пренебречь.

На основании числовых примеров доказано, что при правильном применении деформационной модели, можно устранить выше указанные нестыковки двух нормативных документов. Нестыковки этих задач будут учтены при составлении нового варианта национального норматива AzDTN 2.16-1. 
КЛЮЧЕВЫЕ СЛОВА: нелинейная деформационная модель, диаграмма состояния, нестыковка, длительная прочность бетона, кусочно-линейная форма диаграммы, метод предельных состояний. УДОСКОНАЛЕНА МЕТОДИКА РОЗРАХУНКУ ЗАЛІЗОБЕТОННИХ ЕЛЕМЕНТІВ НА МІЦНІСТЬ I СЕЙСМОСТІЙКІСТЬ З УРАХУВАННЯМ ВИМОГ СВРОКОДІВ

\section{СЕЙФУЛААЕВ ХАНААР КУРБАН ОГАИ.}

Д-р техічний наук, проф., зав. відділом Азербайджанського НДІ будівництва і архітектури, м Баку, Азербайджан

Тел. +994506200710

e-mail: xanlar.seyfullayev@mail.ru

ГАРАЕВ АБДИ НАЗІМ ОГАИ. Канд. техічний наук, директор Азербайджанського НДI будівництва та архітектури,

м Баку, Азербайджан

Тел. +994502152497

e-mail: azimeti.nti@gmail.com

\section{АНОТАЦІЯ}

В роботі розглядається порівняння національного нормативу по залізобетону AzDTN 2.16-1 і однойменного нормативу Франції BAEL-85, виявлено деякі нестиковки результатів розрахунків і вивчені їх причини. Надаються шляхи усунення нестиковки отриманих рішень.

До неузгоджень двох нормативних документів відносяться відмінності в поглядах на граничний стан залізобетонних елементів і результатів розрахунку із застосуванням різних граничних станів, використання нелінійної деформаційної моделі, основою якої $є$ гіпотеза плоских перетинів і діаграми стану бетону, лінеаризації розв'язання задачі шляхом заміни криволінійних форм діаграм стану бетону кусочно-лінійними формами, рішення задачі тривалої міцності бетону з введенням поняття про низхідну гілку криволінійної діаграми деформації бетону та інші проблеми, що характеризують властивості бетону стиснутої зони.

В роботі розглядається статична, геометрична і фізична сторони пружно-пластичного згину залізобетонних елементів. В основу нелінійної деформаційної моделі, i при розрахунку залізобетонних конструкцій, покладені граничні стани залізобетонних елементів, що описуються прямолінійними діаграмами деформації і кусочнолінійними діаграмами стану бетону.

Рішення задачі елементів, що згинаються, на основі нелінійної деформаційної моделі при кусочно-лінійних діаграмах стану бетону, зводиться до вирішення лінійних рівнянь статики, при дотриманні гіпотези плоских перетинів і аналітичних виразів двохлінійної діаграми стану бетону.

На вимогу Еврокодів в розрахунках пропонується використовувати криволінійну діаграму 3 спадаючої гілкою, що відображає поведінку бетону при стисканні. Одночасно, згідно AzDTN 2.16-1, крім кусочно-лінійних діаграм, в розрахунках стану бетону може бути використана криволінійна діаграма 3 спадаючою гілкою. При цьому, повинні бути позначені основні параметричні точки діаграм (максимальне напруження і відповідні деформації, граничні значення і т.д.).

Результати дослідження показують, що при $R_{b l} / R_{b} \geq 0.85$ впливом тривалої міцністі бетону в практичних розрахунках можна знехтувати.

На підставі числових прикладів доведено, що при правильному застосуванні деформаційнної моделі, можна усунути вище зазначені нестиковки двох нормативних документів. Нестиковки цих завдань будуть враховані при складанні нового варіанту національного нормативу AzDTN 2.16-1.

КАЮЧОВІ СЛОВА: нелінійна деформаційна модель, діаграма стану, нестиковка, тривала міцність бетону, кусочно-лінійна форма діаграми, метод граничних станів.

\section{IMPROVED METHODOLOGY OF THE REINFORCED CONCRETE ELEMENTS CALCULATION FOR STRENGTH AND SEISMIC RESISTANCE WITH REGARD TO THE REQUIREMENT OF EUROCODES}

\section{SEYFULLAEV KHANLAR KURBAN OGLI}

Dr.Sci.(Eng), proff., Azerbaijan Scientific-Research Institute of Construction and Architecture,

Baku, Azerbaijan

e-mail: xanlar.seyfullayev@mail.ru

QARAYEV ABDI NAZIM OGLI. Candidate of Technical Sciences, director, Azerbaijan ScientificResearch Institute of Construction and Architecture, Baku, Azerbaijan

Тел. +994502152497

e-mail: azimeti.nti@gmail.com

\section{ABSTRACT}

The paper compares the national standard for reinforced concrete AzDTN 2.16-1 and thee analogous French standard BAEL-85 and identifies some discrepancies in the calculation results with their causes analysis. The ways of eliminating the discrepancy of received solutions are given.

The discrepancies of the two regulations include the different views on the reinforced concrete elements limit state and the calculation results with the use of these different limit states, the use of nonlinear deformation model, the basis of which is the hypothesis of plane sections and concrete state diagrams, the problem solution linearization by replacing the curved shapes of concrete state diagrams with piecewise linear shapes diagrams, 
the solution of the long-term concrete strength problem by introducing the concept of the concrete deformation curvilinear diagram descending branch and other problems characterizing the concrete properties in a compressed zone.

The paper deals with the static, geometrical and physical aspects of the elastic-plastic bending of reinforced concrete elements. The nonlinear deformation model for the reinforced concrete structures calculations is based on the limiting states of reinforced concrete elements, described by the rectilinear strain diagrams and piecewise linear diagrams of concrete state.

The bending elements problem solution on the basis of a nonlinear deformation model with the concrete state piecewise linear diagrams reduces to solving linear equations of statics subject to the hypothesis of flat sections and analytical expressions of a concrete state two-line diagram.

According to the Eurocode requirements, it is proposed to use in the calculations a curved diagram with a descending branch, which reflects the behavior of concrete during compression. At the same time, according to AzDTN 2.16-1, in addition to the piecewise-linear diagrams of concrete state, in the calculations a curved diagram with a descending branch can be used. In this case, the main parametric points of the diagrams (maximum tension and corresponding deformations, boundary values, etc.) must be indicated.

The research results show that at $R_{b l} / R_{b} \geq 0.85$, the influence of the long-term strength of concrete in practical calculations can be neglected.

On the basis of numerical examples it is proved that the deformation model correct application allows eliminating the above discrepancies between the two regulations. The discrepancies of these tasks will be taken into account when drafting a new version of the national standard AzDTN 2.16-1.

KEY WORDS: nonlinear deformation model, state diagram, discrepancy, long-term strength of concrete, piecewise linear shape of the diagram, limit states method.

\section{АКТУААЬНОСТЬ И ПОСТАНОВКА ПРО- БЛЕМЫ}

В 2014 году в газете "Строительная газета" N 19 от 9 мая была опубликовано статья Р. Санжаровского и Т.Мусабаева "Нестыковка актуализированного норматива по железобетону и Еврокода-препятствие в строительстве”. В связи с этим, вопрос был изучен в АзНИИСА и опубликована статья [5].

Следует обратить внимание на то, что создатели метода предельных состояний К.Э.Таль и А.А.Гвоздев [1] неоднократно указывали на несоответствие метода предельных состояний Европейских стран, например BAEL-85 Франции
[4] и метода предельных усилий норматива России СНиП 52.01-2003 [2] и Азербайджана AzDTN 2.16-1 [3].

Сопоставляя национальные и европейские нормы, А.А.Гвоздев указывал на их существенные отличия в принципах и методиках расчета. Устранение этих нестыковок и разработка нового национального норматива по железобетону представляет большой практический интерес и является актуальной проблемой.

\section{АНАЛИЗ ПОСАЕДНИХ ИССАЕДОВАНИЙ И ПУБАИКАЦИЙ}

В работе рассматривается сравнение национального норматива по железобетону AzDTN 2.16-1 и одноименного норматива Франции BAEL-85, выявлены некоторые нестыковки результатов расчетов и изучены их причины. Даются пути устранения нестыковки полученных решений. Эти идеи были приняты и в работе [5]. Сделан вывод о том, что в национальных нормах предельное состояние изгибаемых железобетонных элементов принимается на основании того, что при изгибе железобетонной балки, вследствие развития пластической деформации в арматуре и бетоне, в стадии разрушения наступает предельное состояние, что и определяет разрушающий момент. Но при этом была отвергнута гипотеза плоских сечений и развитие пластической деформации, предполагаемой на основе диаграмм состояний с бесконечной площадкой текучести.

\section{ФОРМУАИРОВАНИЕ ЦЕАЕЙ СТАТЬИ}

Объектом исследования является сравнение результатов расчета железобетонных элементов по национальным и европейским нормам и разработка нового варианта нормы AzDTN 2.16-1.

Предметом исследования является приложение нелинейной деформационной модели к задачам упруго-пластического изгиба железобетонных элементов.

Цель исследования заключается в выявлении причин нестыковки нормативов по железобетону двух нормативных документов AzDTN 2.16-1 и Еврокод-2, указаны пути их устранения.

\section{ИЗАОЖЕНИЕ ОСНОВНОГО МАТЕРИААА}

Прочность железобетонных элементов на основании Еврокода-2 [6] проверяется по предельным значениям деформации сжатого бетона $\varepsilon_{b, u l t}$ и растянутой арматуры $\varepsilon_{s, u l t}$ следующим образом:

$$
\varepsilon_{b, \max } \leq \varepsilon_{b, u l t} ; \quad \varepsilon_{s, \max } \leq \varepsilon_{s, u l t}
$$

Для определения деформации бетона и арматуры с учетом упруго-пластических деформаций материалов используется нелинейная деформационная модель механики твердых деформируемых тел. 
Поэтому, в Европейских странах вместо напряженных состояний железобетонных элементов, предлагается рассматривать диаграммы деформации, полученные на основе гипотезы плоских сечений. Эти состояния приведены ниже (рисунок 2, 3, 4) и в журнале БСТ, Москва, №9, 2017.

Расчетная схема в предельном состоянии получена на основании приложения нелинейной деформационной модели к задачам упругопластического изгиба железобетонных элементом (рисунок 1,b,c).

Диаграммы деформации (плоское сечение) принимаются в виде прямых, проходящих через одну из трех точек, соответствующих предельной деформации бетона и арматуры в диаграммах состояний материалов $\varepsilon_{b 2}, \varepsilon_{s 2}$ и $\varepsilon_{b 0}$. Деформации остальных характерных точек определяются из диаграммы деформаций, а напряжения из диаграмм состояния бетона и арматуры.
I положение. Диаграмма деформации (плоское сечение) проходит через точку А и путем вращения вокруг этой точки получает предельные положения при растяжении. Деформация в точке $A$ равняется $\varepsilon_{s 2}$. Деформации остальных точек бетона, сжатой и растянутой арматуры определяются на основании прямолинейной диаграммы деформации, а затем напряжения в этих точках из диаграммы состояния бетона и арматуры (рисунок 2.).

II положение. Диаграмма деформации проходит через точку В и предельные состояния изгибаемых элементов получаются путем поворота сечений, проходящих через эту точку $B$. Деформация в точке $B$ принимается равной предельной деформации бетон $\varepsilon_{b 2}$. Деформации остальных характерных точек определяются из диаграммы деформаций, а напряжения из диаграмм состояния бетона и арматуры (рисунок 3).

III положение. Диаграмма деформации про-
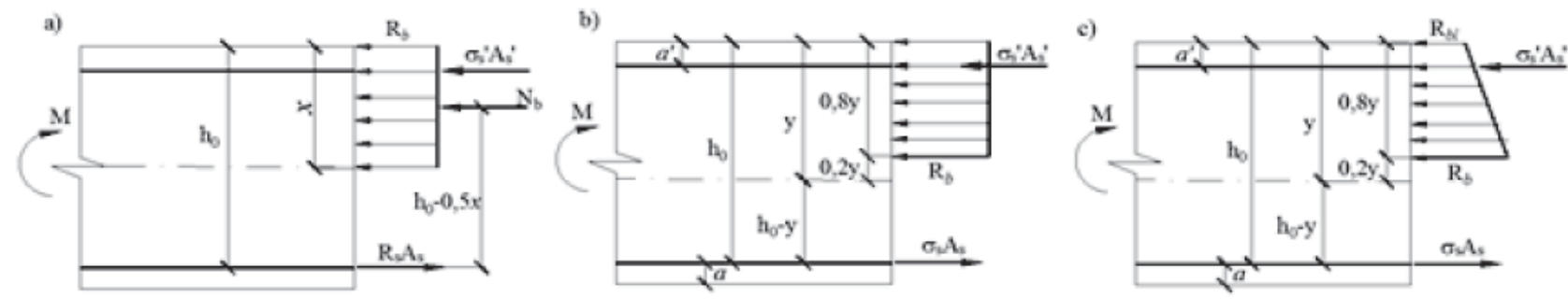

Рис. 1. Предельные состояния изгибаемых элементов а) по СНиП 52.01-2003; b) по Еврокоду; с) с учетом длительной прочности бетона.

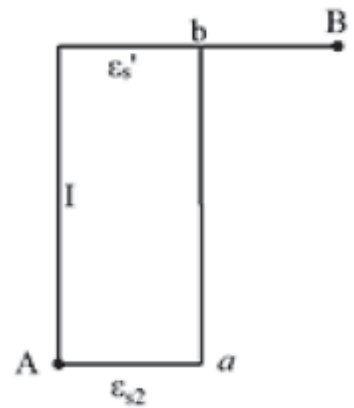

a)

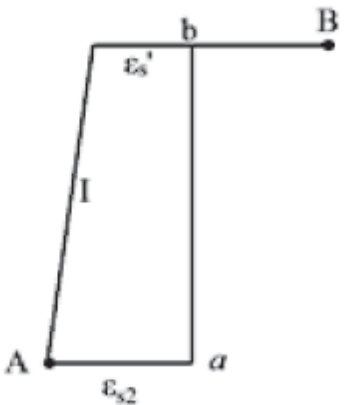

b)

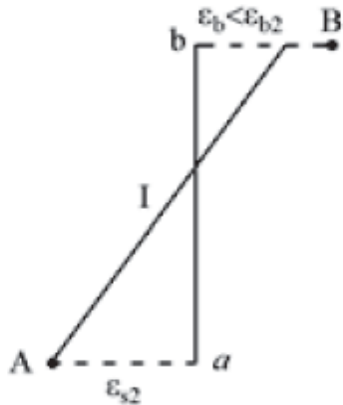

c)

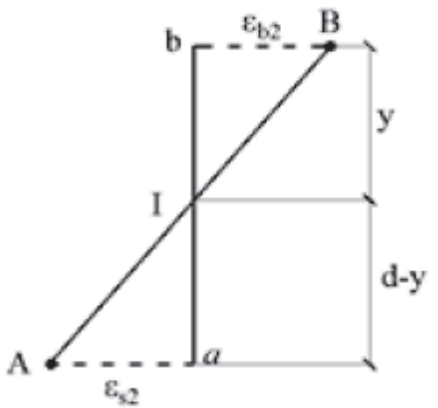

d)

Рис. 2. Диаграмма деформации (предельные состояния при растяжении)

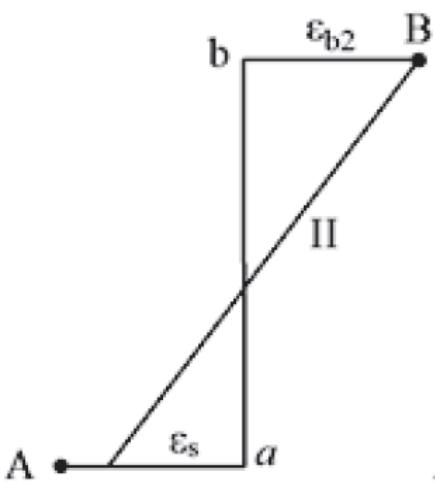

e)

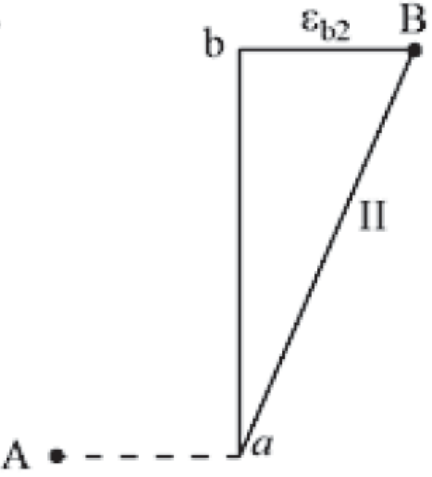

f)

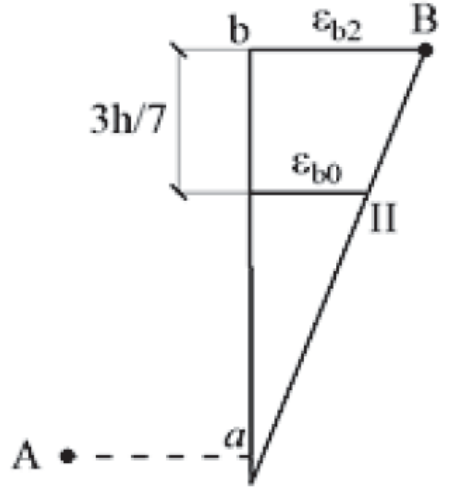

g)

Рис. 3. Диаграммы деформаций при изгибе (предельные состояния) 
ходит через точку $C$, где значение предельной деформации бетона принимается равным $\varepsilon_{b 0}$. Значения деформаций и напряжений характерных точек сечений определяются через деформации бетона $\varepsilon_{b 0}$ (рисунок 4).

Таким образом, геометрическая и физическая стороны задач объединяются в единое и к этим уравнениям присоединяются еще уравнения статики.

Решение задачи в этом варианте соответствует общему правилу решения задач механики твердых деформируемых тел и актуализированный норматив по железобетону надо привести в соответствие с Европейскими стандартами.

Рассматривается статическая, геометрическая и физическая стороны упруго-пластического изгиба железобетонных элементов.

В основу нелинейной деформационной модели при расчете железобетонных конструкций положены предельные состояния железобетонных элементов, описываемые прямолинейными диаграммами деформации и диаграммами состояния бетона.

Уравнения двухлинейной диаграммы бетона принимаем в следующем виде:

$$
\begin{aligned}
& \text { при } 0<\varepsilon_{b}<\varepsilon_{b 1} ; \sigma_{b}=\varepsilon_{b} \cdot E_{b} ; \\
& \text { при } \varepsilon_{b 1}<\varepsilon_{b}<\varepsilon_{b 2} ; \sigma_{b}=R_{b} ; \\
& \text { где } \varepsilon_{b 1}=\frac{R_{b}}{E_{b}} \text { или } E_{b}=\frac{R_{b}}{\varepsilon_{b 1}} .
\end{aligned}
$$

Уравнения трехлинейной диаграммы бетона даны в работе [2].

Решение задачи изгибаемых элементов на основе нелинейной деформационной модели при кусочно-линейных диаграммах состояния бетона сводится к решению следующих уравнений статики при соблюдении гипотезы плоских сечений и аналитических выражений двухлинейной диаграммы состояния бетона (1):

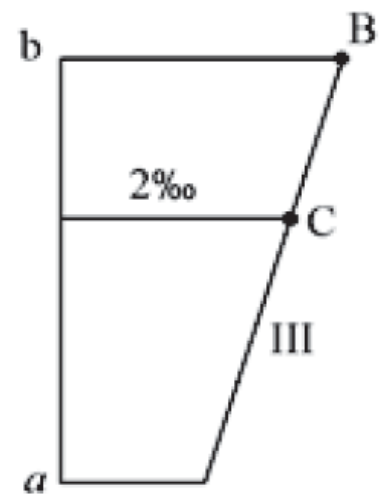

h)

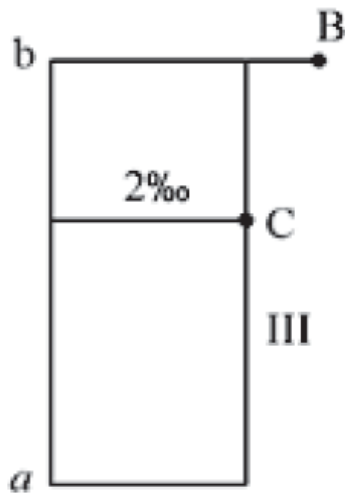

i)

B
Рис. 4. Диаграммы деформации при сжатии (предельные состояния)
1)

$$
\begin{gathered}
M=\int_{A_{b}} \sigma_{b} b y d y+\int_{A_{s}} \sigma_{s}\left(h_{0}-y\right) d A_{s}+\int_{A_{s}^{\prime}} \sigma_{s}^{\prime}\left(y-a^{\prime}\right) d A_{s}^{\prime} \\
\quad \int_{A_{b}} \sigma_{b} b d y-\int_{A_{s}} \sigma_{s} d A_{s}+\int_{A_{s}^{\prime}} \sigma_{s}^{\prime} d A_{s}^{\prime}=0
\end{gathered}
$$

где

$$
\begin{aligned}
& \int_{A_{b}} \sigma_{b} b y d y=\int_{0}^{y_{0}} \varepsilon_{b} \cdot E_{b} b y d y+ \\
& +\int_{y_{0}}^{y} R_{b} b y d y=\frac{R_{b} b y^{2}}{2}\left(1-\frac{1}{3} k_{0}^{2}\right) \\
& \int_{A_{b}} \sigma_{b} b d y=\int_{0}^{y_{0}} \varepsilon_{b} \cdot E_{b} b d y+ \\
& +\int_{y_{0}}^{y} R_{b} b d y=R_{b} b y\left(1-\frac{1}{2} k_{0}\right) .
\end{aligned}
$$

2) линейная диаграмма деформации, полученная на основе гипотезы плоских сечений, где при известном значении предельной деформации бетона $\varepsilon_{b 2}$ определяются деформации арматур $\varepsilon_{s}$ и $\varepsilon_{s}^{\prime}$, а затем напряжения в характерных точках бетона сжатой зоны сечения.

3) кусочно-линейные или криволинейные диаграммы состояния бетона характеризующие деформации и напряжения сжатой зоны бетона.

В результате, объединяются все стороны задачи: статическая, геометрическая (деформационная) и физическая.

В такой постановке с применением нелинейной деформационной модели к задачам об изгибе железобетонных элементов на основе нового понятия о предельном состоянии получен классический метод расчета по предельному состоянию и различие взглядов на предельные состояния между российским и европейским нормативами снимаются.

Решение задачи принимает вид:

$$
\left\{\begin{array}{l}
M=\frac{R_{b} b y^{2}}{2}\left(1-\frac{1}{3} k_{0}^{2}\right)+\sigma_{s} A_{s}\left(h_{0}-y\right)+ \\
+\sigma_{s}^{\prime} A_{s}^{\prime}\left(y-a^{\prime}\right) ; \\
R_{b} b y\left(1-\frac{1}{2} k_{0}\right)-\sigma_{s} A_{s}+\sigma_{s}^{\prime} A_{s}^{\prime}=0 .
\end{array}\right.
$$

Здесь $k_{0}$ - коэффициент, характеризующий уровень пластических деформаций сжатой зоны бетона, и в зависимости от $k_{0}$ определяются напряженные состояния изгибаемых железобетонных элементов:

а) $k_{0}=1$. В этом случае пластические деформации не появляются и изгибаемый элемент работает в стадии упругого состояния. Расчет сводится к известному методу допускаемых напряжений (рисунок.6,a). 
b) $0,2 \leq k_{0} \leq 1$. В этом случае сжатая зона бетона разбита на упругую и пластическую зоны (рисунок $6, \mathrm{~b})$.

c) $k_{0} \leq 0,2$. В этом случае около нейтрального слоя $y_{0} \leq 0,2 y$ упругие деформации незначительные и можно ими пренебречь. Расчет сводится к методу предельных состояний и эпюра напряжения в бетоне прямоугольная (рисунок 6,с). Это решение полностью совпадает с методом предельных состояний, приведенных в Еврокодах.

Рассматривается другой новый и практический подход решения задачи.

В решение задачи введены следующие новые понятия: $y_{0} \leq k_{0} y$

где $k_{0}=\frac{\varepsilon_{b 1}}{\varepsilon_{b, \max }}$ и $\varepsilon_{b}=\varepsilon_{b 1} \frac{y}{y_{0}}$.

Найдена граница между упругой и пластической областями сжатой зоны бетона (рисунок 5).

Такой подход вводится для того, чтобы разработать новый механизм построения эпюр нормальных напряжений в сжатой зоне бетона и свести решение задачи к известному методу предельных состояний.

Исходя из определения железобетона, для полного использования прочности бетона сжатой зоны относительная деформация в крайних волокнах сжатой зоны бетона должна быть равной $\varepsilon_{b 2}$, независимо от метода расчета железобе- тонных элементов. Исходя из этой идеи о железобетоне можно предложить механизм построения эпюры напряжений в сжатой зоне бетона изгибаемых элементов. Этот механизм построения эпюр, нормальных напряжений в бетоне сжатой зоны сечения, основан на совместном приложении к прямолинейной диаграмме деформации и диаграмме состояния бетона, выполняется на основании диаграммы состояния бетона, переход из деформированного состояния к напряженному осуществляется по следующему правилу:

в прямолинейной диаграмме деформации сечения находится точка $k_{0}$, соответствующая упругой деформации $\varepsilon_{b l}$ на расстоянии $y_{0}$ от нейтральной оси.

В области, которая находится на расстоянии $y_{0}=k_{0} y$, напряжение изменяется по закону $\sigma_{b}=\varepsilon_{b} \cdot E_{b}$, а в точках $\varepsilon_{b}>\varepsilon_{b 1}$ бетон находится в пластической области и напряжение в бетоне будет $\sigma_{b}=R_{b}=$ const.

Таким образом, эпюра напряжений в сжатой зоне бетона принимает вид, указанный на рисунке 5 a,b.

В зависимости от коэффициента $k_{0}$ в расчетах на основе нелинейной деформационной модели эпюры, напряжения в бетоне принимают три основные формы (рисунок 6).

После построения эпюр нормальных напряжений, которые являются объединением геометри-
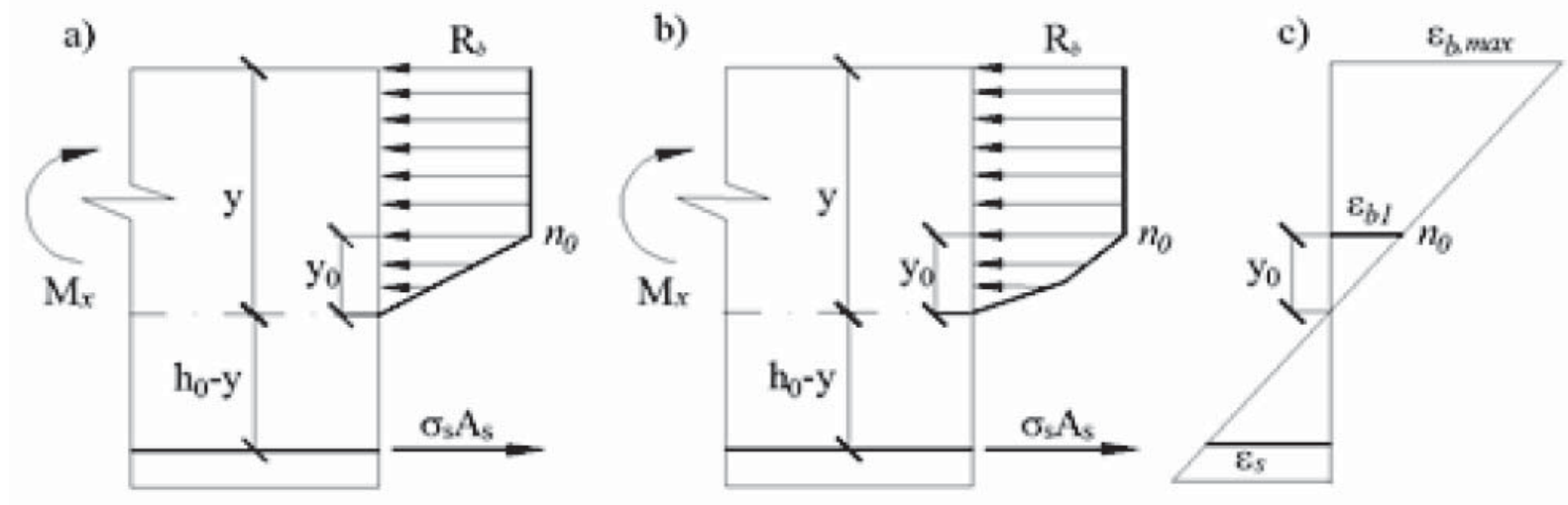

Рис. 5. Расчетная схема, полученная на основе кусочно-линейных диаграмм состояния бетона: a) при двух; b) при трех
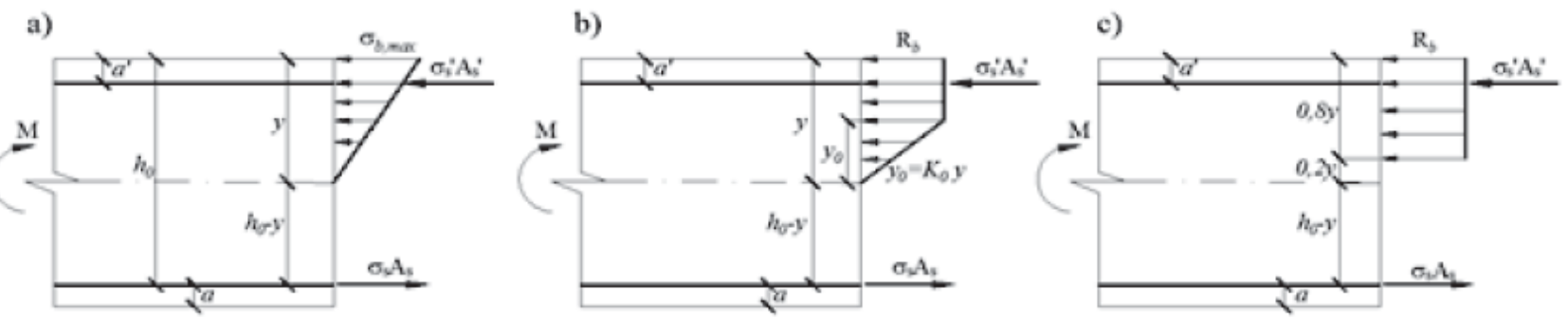

Рис. 6. Три стадии напряженного состояния изгибаемых элементов 
ческой (деформационной) и физической (диаграммы состояния бетона) стороны задачи, рассматривается равновесие элемента (статическая сторона). Таким образом, решение задачи получено по правилу механики твердых деформируемых тел.

Предложенным вариантом расчета изгибаемых элементов, на основе нелинейной деформационной модели, есть метод расчета по предельным состояниям и является самым простым и доступным.

Поэтому, этот вариант расчета считается усовершенствованием метода предельных состояний. По нашему мнению, этим руководствовались и создатели норматива по железобетону европейских стран [4]. Здесь нет необходимости решать нелинейные алгебраические уравнения методом итерации, которые требуют доказательства их сходимости, точности решения и возможность определения несущей способности железобетонных элементов $[1,7]$.

Этим доказано, что метод расчета, по предельным состояниям железобетонных элементов, есть приложение способа решения задачи механики твердых деформируемых тел и результаты расчета полностью подтверждаются экспериментами проводимыми под руководством А.А. Гвоздева [10].

Таким образом, нестыковки по двум нормативам снимаются.

В методике расчета изгибаемых железобетонных элементов, по предельным состояниям, также имеются некоторые различия. Если в нормативе СНиП 52.01-2003, в расчетах по предельному состоянию, исходят из стадии разрушения, где напряжение в сжатой зоне бетона $R_{b}$ и в растянутой арматуре принимаются равным $R_{s}$, что в некоторых случаях приводят к неопределенностям, например при внецентренном сжатии и предварительно-напряженных элементах, при малых әксцентриситетах с использованием эмпирической формулы, а в расчетах по Еврокоду относительная деформация наиболее сжатых волокон принимается известным и равным $\varepsilon_{b 2}$, а через нее определяются остальные неизвестные деформации и напряжения на основе гипотезы плоских сечений и кусочно-линейных диаграмм состояния бетона и арматуры и нет необходимости использования эмпирической формулы для определения $\sigma_{s}[5]$.

Нестыковка нормативов по железобетону России и европейских стран объясняется в неправильном применении нелинейной деформационной модели к задачам изгиба железобетонных элементов [7].

Жесткость изгибаемых железобетонных элементов с учетом упруго-пластической деформации определяется следующим образом:

$$
D=M \frac{y}{\varepsilon_{b, \max }}
$$

где $M$ определяется по формуле (3), тогда

$$
D=\frac{R_{b}}{\varepsilon_{b, \max }}\left[\begin{array}{l}
\frac{b y^{3}}{2}\left(1-\frac{1}{3} k_{0}^{2}\right)+\frac{\sigma_{s}}{R_{b}} A_{s} y\left(h_{0}-y\right)+ \\
+\frac{\sigma_{s}^{\prime}}{R_{b}} A_{s}^{\prime} y\left(y-a^{\prime}\right)
\end{array}\right] .
$$

Эта формула справедлива для любого значения коэффициента $k_{0}=0,15 \div 1$, но значения напряжений в растянутых и сжатых арматурах $\sigma_{s}$ и $\sigma_{s}^{\prime}$ зависят от напряженных состояний изгибаемых элементов и они определяются следующим образом.

Сначала находим $\varepsilon_{s}=\varepsilon_{b 1} \frac{h_{0}-y}{y_{0}}$ и $\varepsilon_{s}^{\prime}=\varepsilon_{b 1} \frac{y-a^{\prime}}{y_{0}}$.

Если $\varepsilon_{s} \leq \varepsilon_{s l}$ тогда $\sigma_{s}=\varepsilon_{s} \cdot E_{s}$.

Если $\varepsilon_{s}>\varepsilon_{s I}$ тогда $\sigma_{s}=R_{s}$.

В случае $\varepsilon_{s} \leq \varepsilon_{s l}$ :

$\frac{\sigma_{s}}{R_{b}}=\frac{E_{s} \varepsilon_{s}}{E_{b} \varepsilon_{b 1}}=n \frac{\varepsilon_{b 1}}{\varepsilon_{b 1}} \frac{h_{0}-y}{y_{0}}=n \frac{h_{0}-y}{y_{0}}=\frac{n}{k_{0}} \frac{h_{0}-y}{y}$

и $\frac{\sigma_{s}^{\prime}}{R_{b}}=\frac{n}{k_{0}} \frac{y-a^{\prime}}{y} \quad \frac{R_{b}}{\varepsilon_{b, \max }}=\frac{E_{b} \varepsilon_{b 1}}{\varepsilon_{b, \max }}=E_{b} \cdot k_{0}=E_{b}^{\prime}$

$$
\frac{R_{s}}{R_{b}}=\frac{n}{k_{0}} \cdot \frac{h_{0}-y}{y},
$$

тогда

$$
D=E_{b}^{\prime}\left[\begin{array}{l}
\frac{b y^{3}}{2}\left(1-\frac{1}{3} k_{0}^{2}\right)+\frac{\sigma_{s}}{R_{b}} A_{s} y\left(h_{0}-y\right)+ \\
+\frac{\sigma_{s}^{\prime}}{R_{b}} A_{s}^{\prime} y\left(y-a^{\prime}\right)
\end{array}\right] .
$$

В упругой стадии жесткость имеет вид $\left(k_{0}=1\right)$

$$
\begin{gathered}
D=E_{b} \cdot I_{x, \text { red }} \\
I_{x, \text { red }}=\frac{b y^{3}}{3}+n \cdot A_{s}\left(h_{0}-y\right)^{2}+n \cdot A_{s}^{\prime}\left(y-a^{\prime}\right)^{2} .
\end{gathered}
$$

В остальных случаях $k_{0} \neq 1$, имеем:

$$
\frac{\sigma_{s}}{R_{b}}=\frac{R_{s}}{R_{b}}=\frac{n}{k_{0}} \cdot \frac{h_{0}-y}{y} \text { и } D=E_{b}^{\prime} \cdot I_{x, \text { red }}^{*} ;
$$

где:

$$
\begin{aligned}
& I_{x, \text { red }}^{*}=\frac{b y^{3}}{2}\left(1-\frac{1}{3} k_{0}^{2}\right)+\frac{n}{k_{0}} A_{s}\left(h_{0}-y\right)^{2}+ \\
& +\frac{n}{k_{0}} \cdot A_{s}^{\prime}\left(y-a^{\prime}\right)^{2} .
\end{aligned}
$$

Или

$$
D=k_{b} E_{b} I_{b}+k_{s} E_{s} I_{s}
$$


здесь:

$$
\begin{aligned}
& k_{b}=1,5 k_{0}\left(1-\frac{1}{3} k_{0}^{2}\right) ; \\
& k_{s}=1 ; \quad I_{b}=\frac{b y^{3}}{3} ; \\
& I_{s}=A_{s}\left(h_{0}-y\right)^{2}+A_{s}^{\prime}\left(y-a^{\prime}\right)^{2} .
\end{aligned}
$$

Относительная деформация бетона наиболее сжатых волокон сечения определяется по следующей формуле:

$$
\varepsilon_{b, \max }=\frac{M \cdot y}{D} .
$$

Условие прочности в соответствии п.8.1.24 СНиП 52.01-2003 проверяется следующим обра3ом:

$$
\left|\varepsilon_{b, \max }\right| \leq \varepsilon_{b 2}
$$

Рассматриваются следующие варианты расчета: a) $\varepsilon_{b, \max } \leq \varepsilon_{b 1} ; \quad$ b) $\varepsilon_{b 1}<\varepsilon_{b, \max }<\varepsilon_{b 2} ; \quad$ c) $\varepsilon_{b, \max }=\varepsilon_{b 2}$

Эпюры напряжений при расчетных вариантах показаны на рисунке 6 .

Если в расчетах исходили бы из идеи о железобетоне $\varepsilon_{b, \max }=\varepsilon_{b 2}$, тогда бы получили предельное состояние железобетонного элемента (рисунок 6,c).

По требованию Еврокода в расчетах предлагается использовать криволинейную диаграмму с ниспадающей ветвью, отражающую поведение бетона при сжатии. Одновременно, согласно СНиП 52.01 -2003, кроме кусочно-линейных диаграмм состояния бетона в расчетах может быть использована криволинейная диаграмма с ниспадающей ветвью. При этом, должны быть обозначены основные параметрические точки диаграмм (максимальные напряжения и соответствующие деформации, граничные значения и т.д.).

Криволинейная диаграмма состояния бетона, определяющая связь между напряжениями и относительными деформациями дана в СНиП
$52.01-2003$.

Согласно опытным данным, при длительном действии нагрузки под влиянием развивающихся значительных неупругих деформаций и структурных изменений бетон разрушается при напряжениях, меньших, чем временное сопротивление бетона осевому сжатию $R_{b}$. Предел длительного сопротивления бетона сжатию, по опытным данным, может составлять $R_{b l}=0,9 R_{b}$ и меньше. Если, при эксплуатации конструкции, в благоприятных для нарастания прочности бетона условиях, уровень напряжений $\sigma_{b} / R_{b l}$ постепенно уменьшается, то отрицательное влияние фактора длительного нагружения может и не наблюдается (рис. 7) [10].

При сжатии бетонной призмы, в режиме пропорционального развития во времени продольных деформаций обнаруживается постоянное снижение сопротивления бетона, так называемая ниспадающая ветвь диаграммы напряжениядеформации (рисунок 7). Такой участок повышенного деформирования бетона реально наблюдаются в конструкциях при определенных условиях нагружения, например при сжатии бетона у внешней грани сжатой зоны изгибаемых элементов. При длительном действии нагрузки, неупругие деформации бетона с течением времени увеличиваются. Наибольшая интенсивность нарастания неупругих деформаций наблюдается первые 3-4 месяца и может продолжаться несколько лет.

На диаграмме (рисунок 7,a) участок $O A$ характеризует деформации, возникающие при нагружении, участок АВ характеризует уменьшение прочности бетона при длительном действии нагрузки, участок $A B_{1}$ характеризует нарастание неупругих деформаций при постоянном значении напряжений, участок $A B_{2}$ характеризует нарастание прочности бетона в течении длительного времени.

В изгибаемых элементах, в соответствии с диаграммой состояния бетона с ниспадающей ветвью, эпюра напряжения бетона сжатой зоны показана на рисунок 7,b. Криволинейная диаграмма состояния бетона заменяется двухлинейной диаграммой.
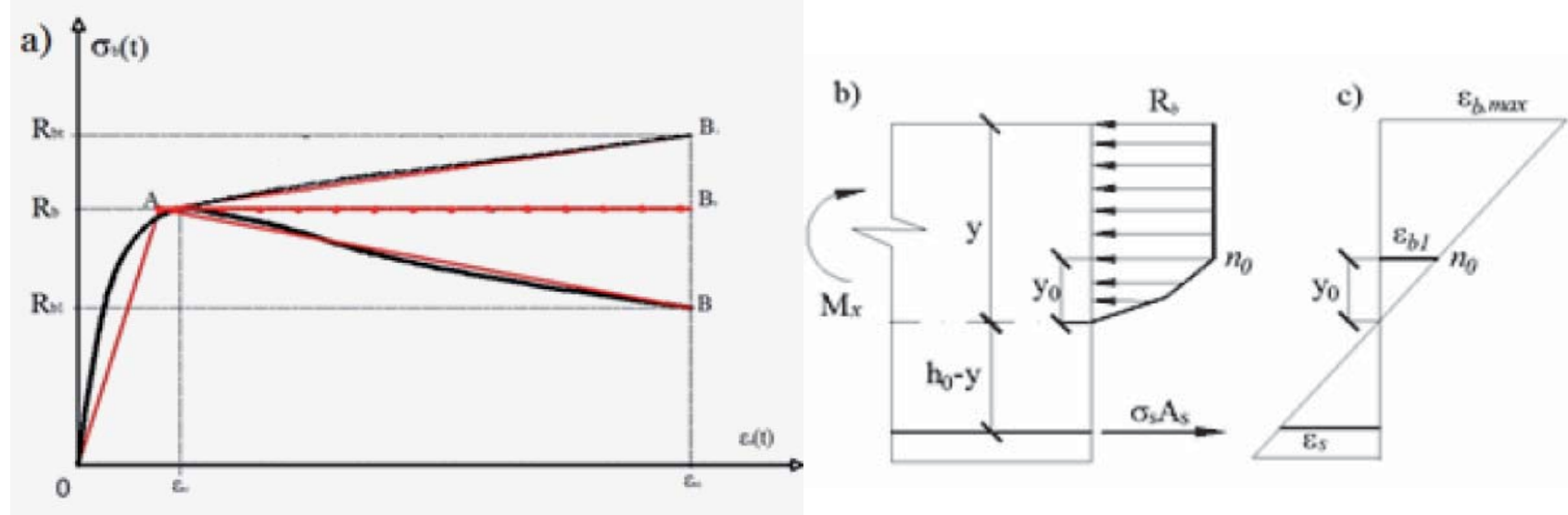

Рис. 7. Расчетная схема изгибаемых элементов на основе криволинейной диаграммы бетона с ниспадающей ветвью 
Аналитические выражения двухлинейной диаграммы состояния бетона с ниспадающей ветвью принимают следующий вид:

при $0<\varepsilon_{b}<\varepsilon_{b 1}$ тогда $\sigma_{b}=\varepsilon_{b} \cdot E_{b}$.

При

$\varepsilon_{b 1} \leq \varepsilon_{b} \leq \varepsilon_{b 2}$

$\sigma_{b}=R_{b}\left[\left(1-\frac{\varepsilon_{b}-\varepsilon_{b 1}}{\varepsilon_{b 2}-\varepsilon_{b 1}}\right)+\frac{R_{b l}}{R_{b}} \frac{\varepsilon_{b}-\varepsilon_{b 1}}{\varepsilon_{b 2}-\varepsilon_{b 1}}\right]$.

Где $\varepsilon_{b 1}=\frac{R_{b}}{E_{b}}$.

$R_{b l}$ - длительная прочность бетона.

На основании гипотезы плоских сечений находим: $\varepsilon_{b}=\varepsilon_{b 2} \cdot \frac{y_{1}}{y}$.

Как показали исследования, приложения нелинейной деформационной модели к задачам изгиба железобетонных элементов в конечном итоге приводит к построению эпюры напряжений в сжатой зоне бетона, механизм которого демонстрирован выше. В предельном состоянии схема показана на рисунок 8,a.

Условия расчета изгибаемых элементов на прочность составляются относительно центра тяжести растянутой арматуры в следующем виде:

$$
\left\{\begin{array}{l}
M \leq R_{b} b h_{0}^{2} \xi\left(1-k_{0}\right)\left[1-\frac{\xi}{2}\left(1-k_{0}\right)\right]- \\
-\frac{1}{2} R_{b l}^{*} b h_{0}^{2} \xi\left(1-k_{0}\right)\left[1-\frac{\xi}{3}\left(1-k_{0}\right)\right]+ \\
+\frac{1}{2} R_{b} b h_{0}^{2} \xi\left[1-\xi+\frac{2}{3} k_{0} \xi\right]+\sigma_{s}^{\prime} A_{s}^{\prime}\left(h_{0}-a^{\prime}\right) ; \\
R_{b} b h_{0} \xi\left(1-k_{0}\right)-\frac{1}{2} R_{b l}^{*} b h_{0} \xi\left(1-k_{0}\right)+ \\
+\frac{1}{2} R_{b} b h_{0} k_{0} \xi+\sigma_{s}^{\prime} A_{s}^{\prime}-\sigma_{s} A_{s}=0 .
\end{array}\right.
$$
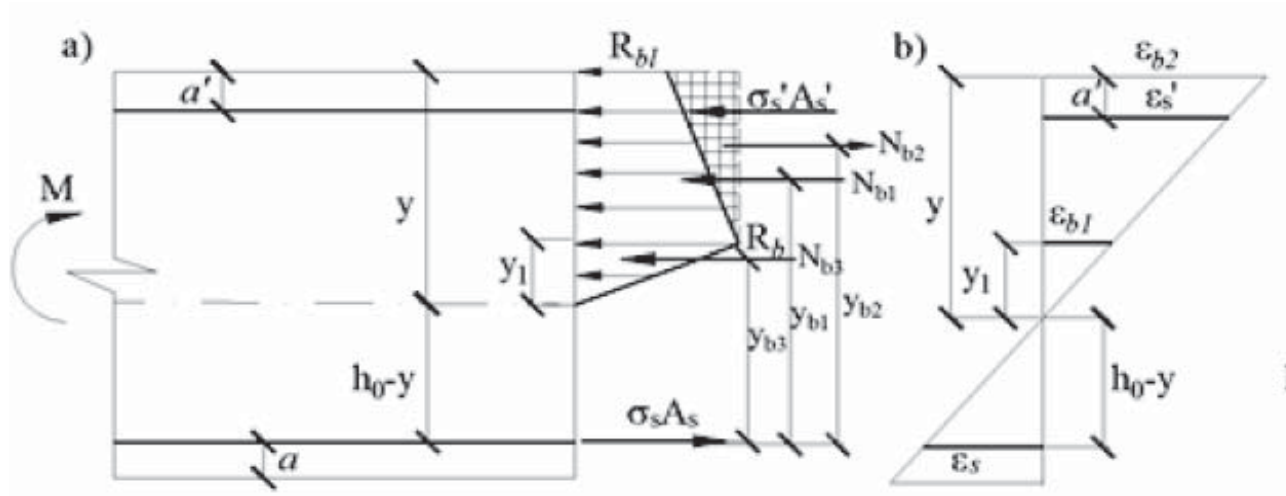

$$
M_{u}=A_{R} R_{b} b h_{0}^{2}
$$

$$
\xi_{R}=\frac{1}{1+\frac{\varepsilon_{s 1}}{\varepsilon_{b 1}}}=\frac{1}{1+\frac{0,00175}{0,0035}}=0,667
$$

Значение предельных моментов с учетом всей диаграммы деформированного состояния бетона имеют вид:

Сначала рассмотрим введение в расчет эпюры сжимающих напряжений, когда в (12) не учитывается длительная прочность бетона при $R_{b l}=R_{b}$ имеем:

$$
A_{R}=0,391+0,0281=0,419 .
$$

Пренебрегая влиянием упругих деформации в близи нейтрального слоя изгибаемых элементов, получим:

$$
A_{R}=0,391 \text {. }
$$

Рис. 8. Расчетная схема элементов с учетом длительной прочности бетона 
Таким образом, в предельном состоянии различие между двумя решениями (с учетом всей диаграммы состояния бетона и только ниспадающего участка) будет в пределах 6,7\%. В этом случае, расчетная схема принимается в виде рисунка 1в.

Влияние длительной прочности бетона, с использованием всей диаграммы длительного деформирования имеет такой вид:

при $R_{b l}=R_{b} ; \quad A_{R}=0,419$

при $R_{b l}=0,9 R_{b} ; \quad A_{R}=0,397$

при $R_{b l}=0,85 R_{b} ; \quad A_{R}=0,383$

при $R_{b l}=0,8 R_{b} . \quad A_{R}=0,375$

Как показывают результаты численных примеров, учет длительной прочности $R_{b l}$ мало влияет на результаты расчета и практически ими можно пренебречь.

Рассмотрим вопрос об учете длительной прочности бетона в расчете изгибаемых элементов с диаграммой состояния бетона с ниспадающего участка. Известно, что в предельном состоянии при $y_{0} \leq 0,2 y$ или $k_{0} \leq 0,2$ упругие деформации вблизи нейтрального слоя незначительные и пренебрегая ими расчетная схема имеет следующий вид (рисунок 1с и 1,9 a).

Эпюра напряжения в сжатой зоне бетона с учетом длительной прочности бетона принимается в виде трапеции. Тогда уравнения равновесия имеют вид:

$$
\begin{aligned}
& M \leq 0,8 R_{b} b y\left(h_{0}-0,4 y\right)-0,4 b y\left(R_{b}-R_{b l}\right) \times \\
& \times\left(h_{0}-0,267 y\right)+\sigma_{s}^{\prime} A_{s}^{\prime}\left(h_{0}-a^{\prime}\right) ; \\
& 0,8 R_{b} b y\left(h_{0}-0,4 y\right)-0,4 b y\left(R_{b}-R_{b l}\right)+ \\
& +\sigma_{s}^{\prime} A_{s}^{\prime}-\sigma_{s} A_{s}=0 .
\end{aligned}
$$

Если пренебречь длительной прочностью бетона, т,е. $R_{b l}=R_{b}$, получим известные решения пре-

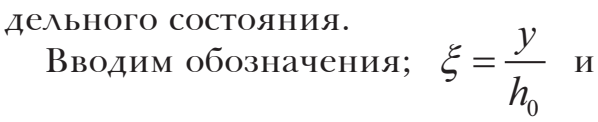

$$
A_{0}=0,8 \xi(1-0,4 \xi)-0,4 \xi(1-0,267 \xi)\left(1-\frac{R_{b l}}{R_{b}}\right)
$$

$$
\xi_{R}=\frac{1}{1+\frac{\varepsilon_{s 1}}{\varepsilon_{b 1}}}=\frac{1}{1+\frac{0,00175}{0,0035}}=0,667
$$

Тогда получим:

$A_{R}=0,8 \xi_{R}\left(1-0,4 \xi_{R}\right)-0,4 \xi_{R}\left(1-0,267 \xi_{R}\right)\left(1-\frac{R_{b l}}{R_{b}}\right)$.

В примере также учитывается нарастание прочности бетона на портландцементе при положительной температуре твердения $\left(15^{\circ} \mathrm{C}\right)$ и влажной среде, определяемые формулой [10]:

$$
R_{b t}=0,7 R_{b} \lg t
$$

$R_{b l}$ - временное сопротивление сжатию бетона в возрасте $t$ суток.

$R_{b t=60}=1,245 R_{b} ; \quad R_{b t=90}=1,368 R_{b} ;$

$R_{b t=120 \partial}=1,450 R_{b b} ; \quad R_{b t=1}=1,79 R$.

Если учесть уменьшение длительной прочности бетона $R_{b l} \geq 0,85 R_{b}$ во времени, а также нарастания прочности бетона во времени $R_{b l} \approx 1,3 R_{b}$, тогда можно установить, что на диаграмме состояния бетона ниспадающая ветвь не образуется, следовательно $R_{b l}=R_{b}$ и расчет ведется без учета $R_{b l}$.

Несущая способность изгибаемых железобетонных элементов определена по формуле (13): $M_{u}=A_{R} R_{b} b h^{2}{ }_{0}$, и получины следующие значения Для $A_{R}$ :
1) $A_{R}=0,391$
2) $A_{R}=0,369$;
3) $A_{R}=0,360$
4) $A_{R}=0,351$

Результаты исследования показывают, что при $\frac{R_{b l}}{R_{b}} \geq 0,85$ влиянием длительной прочности бетона в практических расчетах можно пренебречь.

На основании теоретических результатов исследования и численных примеров можно сделать следующие выводы.
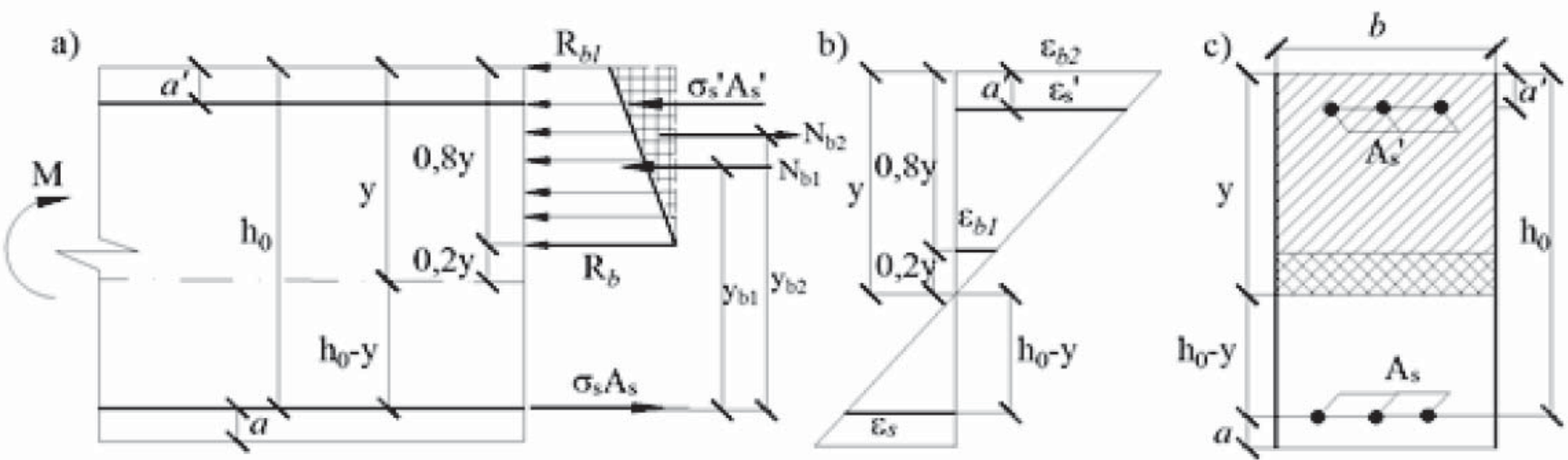

Рис. 8. Расчетная схема изгибаемых элементов по предельному состоянию с учетом длительной прочности бетона 


\section{ВЫвОдЫ}

1. Доказано, что нелинейная деформационная модель является общим правилом решения задач механики твердых деформируемых тел и она в Европейских странах служила для создания метода расчета железобетонных элементов по предельному состоянию. Правильное применение ее к задачам упруго-пластического изгиба железобетонных элементов сводится к известному методу расчета по предельным состояниям Еврокода.

2. Установлено, что при использовании диаграмм состояния бетона в виде кусочнолинейных форм, при интегрировании уравнения статики, некоторые авторы допустили ошибки, в результате чего вместо линейных алгебраических уравнений получены нелинейные, приводящие к неверным результатам, которые не подтверждаются ни математически, ни экспериментально.

3. Установлено, что при использовании диаграммы состояния бетона с ниспадающей ветвью, учетом влияния длительной прочности бетона в практических расчетах железобетонных элементов можно пренебречь.

\section{БИБАИОГРАФИЧЕСКИЙ СПИСОК}

1. Строительная газета. Нестыковка актуализированного норматива по железобетону и Еврокода - препятствие в строительстве. №19. 9 мая, 2014.

2. СНиП 52-01-2003 Бетонные и железобетонные конструкции. Основные положения. М. 2012.

3. AzDTN 2.16-1 Бетонные и железобетонные конструкции. Нормы проектирования, Баку. 2015. 131 с.

4. Regles techniques de conception et de calcul des ouvrages et constructions en beton armé (BAEL-83), Paris, 1983.

5. Сейфуллаев Х.К., Гараев А.Н., О нестыковке национальных нормативов по железобетону и Еврокодов, БСТ. №9. 2017. С. 40-45.

6. Биби Э.В., Нараянан Р.С. Руководство для проектировщиков к Еврокоду 2, Москва, МГСУ. 2013. - 292 с.

7. Гаджиев М. А. Прочность и устойчивость железобетонных стержневых элементов с применением нелинейных диаграмм деформирования материалов при кратковременном и длительном загружениях: Автореферат докторской диссертации, Баку. 2007.

8. Charon Pierre. Calcul des ouvrages en beton armé suivant les régles BAEL - 83. Théorie et aplication, Paris, Eyrolles. 1986. 460 p.
9. M.Rosh. Lé stabilité des barres comprimées par des forces excentrées. Paris. 1932.

10. Байков В.Н., Сигалов Э.С. Железобетонные конструкции М,Стройиздат. 1991. 767с.

\section{REFERENCES}

1. Discrepancy between the national standard for reinforced concrete and Eurocode is an impediment to construction (2014). Stroitelnaia gazeta, 19 (May 9) [in Russian].

2. Concrete and reinforced concrete structures. Main provisions. (2012). SNiP 52-01-2003. Moscow [in Russian].

3. Concrete and reinforced concrete structures. Design standards. (2015). AzDTN 2.16-1. Baku [in Russian].

4. Regles techniques de conception et de calcul des ouvrages et constructions en beton armé (BAEL-83). (1983). Paris [in French).

5. Seifullaiev, K.K. \& Garaiev, A.N. (2017). On discrepancies between national standards for reinforced concrete and Eurocodes. (2017). BST, 9, 40-45 [in Russian].

6. Beeby,A.W.\& Narayanan, R.S. Designers' Guide to Eurocode 2: Design of concrete structures. (2013). (V.O. Almazov \& A.I. Plotnikov, Eds.). Moscow: MSUCE [in Russian].

7. Gadzhiev, M. A. (2007). Strength and stability of reinforced concrete rod elements with the application of nonlinear diagrams of materials deformation under short-term and longterm loadings. Doctor's (Candidate's) thesis / (Extended abstract of Doctor's (candidate's) thesis). Baku [in Russian].

8. Charon, P. (1986). Calcul des ouvrages en beton armé suivant les régles BAEL - 83 . Théorie et application. Paris: Eyrolles [in French).

9. Rosh, M. (1932). Le stabilité des barres comprimées par des forces excentrées. Paris [in French).

10. Baikov, V.N. \& Sigalov, E.S. (1991). Reinforced concrete structures. Moscow: Stroiizdat [in Russian].

Статья поступила в редакцию 02.04.2019 года 